\title{
CONCEPTS FOR THE JLAB AMPERE-CLASS CW CRYOMODULE *
}

\author{
R. Rimmer, E.F. Daly, W.R. Hicks, J. Henry, J. Preble, M. Stirbet, H. Wang, K.M. Wilson, G. Wu, \\ JLab, Newport News, VA 23606, USA
}

\section{Abstract}

We describe the concepts and developments underway at JLab as part of the program to develop a new CW cryomodule capable of transporting Ampere-level beam currents in a compact FEL. Requirements include realestate gradient of at least $10 \mathrm{MV} / \mathrm{m}$ and very strong $\mathrm{HOM}$ damping to push BBU thresholds up by two or more orders of magnitude compared to existing designs. Cavity shape, HOM damping, power couplers, tuners etc. are being designed and optimized for this application. Cavity considerations include a large iris for beam halo, low-RF losses, HOM frequencies and Q's, low peak surface fields, field flatness and microphonics. Module considerations include high packing factor, low static heat leak, image current heating of beam-line components, cost and maintainability. This module is being developed for the next generation ERL based high power FELs but may be useful for other applications such as electron cooling, electron-ion colliders, industrial processing etc.

\section{INTRODUCTION}

High-power FELs and other machines based on high current energy recovered linacs face many of the same issues as high-current storage rings, including beam heating of components and beam break-up (BBU) instabilities. For the next generation compact FEL being developed at JLab a new cryomodule is required that is capable of accelerating and energy recovering up to Ampere levels of beam current. This requires raising the BBU threshold by at least two orders of magnitude compared to existing designs. Table 1 gives a summary of the proposed specifications for the new module. To achieve these goals we propose to use a compact waveguide-damped multi-cell cavity packaged in an SNSstyle cryomodule. Challenges include extreme HOM damping, high HOM power and high fundamental-mode power (in operating scenarios where energy recovery is designed to be less than $100 \%$ ).

Table 1: FEL Ampere-class module draft specifications.

\begin{tabular}{|c|c|}
\hline Voltage & $100-120 \mathrm{MV}$ \\
\hline Length & $\sim 10 \mathrm{~m}$ \\
\hline Frequency & $750 \mathrm{MHz}$ \\
\hline Beam Aperture & $>3$ ' $(76.2 \mathrm{~mm})$ \\
\hline BBU Threshold & $>1 \mathrm{~A}$ \\
\hline HOM Q's & $<10^{4}$ \\
\hline Beam power & $0-1 \mathrm{MW}$ \\
\hline
\end{tabular}

* This manuscript has been authored by SURA, Inc. under Contract No. DE-AC05-84ER-40150 with the U.S. Department of Energy, and by The Office of Naval Research under contract to the Dept. of Energy.

\section{CRYOMODULE COMPONENTS}

Where possible components are based on tried and tested designs, however some items have to be new or heavily modified because of the demanding application.

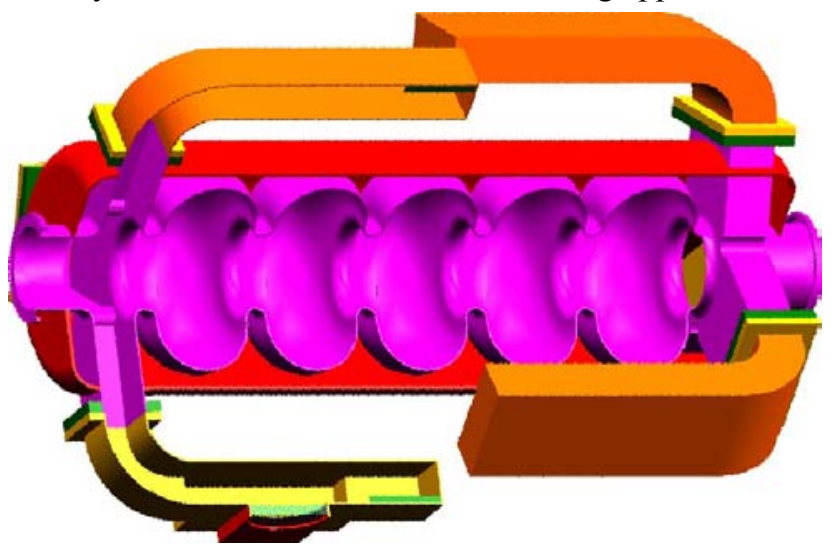

Figure 1: Cavity section with rounded pillbox profile, waveguide HOM dampers and waveguide window.

\section{General Layout}

The overall layout is based on the JLab space-frame design. Six 5-cell cavities are sufficient to provide the desired voltage, assuming an average performance of 16.7 MV/m, which is similar to that of the SNS high-beta cavities. Each cavity has its own helium vessel that also cools the end groups. There are no helium-to-beam-line vacuum flanged joints. HOM power is taken out to ambient temperature by waveguide dampers with watercooled HOM loads. There are no bellows between cavities and beam-line thermal contraction is accommodated by warm bellows at the ends of the module as on the JLab 12 $\mathrm{GeV}$ upgrade design.

\section{Cavity shape}

We propose to use five cells per cavity which is a good compromise between packing factor, HOM damping efficiency, and facilities constraints. This design meets the HOM Q and impedance requirements while maintaining a healthy real-estate gradient [1]. A rounded pillbox cell shape, figure 1, gives excellent fundamental mode efficiency, strong HOM damping and places HOM frequencies safely between dangerous harmonics of the bunch frequency. A study of many different cell shapes [2], suggests that this is a good compromise between HOM power and operating mode efficiency. The large $(140 \mathrm{~mm})$ iris diameter gives good clearance for beam halo, strong cell to cell coupling and good HOM damping. Stiffening rings may be added to ensure that microphonic resonances are kept at suitably high frequencies. 


\section{HOM damping}

While there are many methods of HOM damping we believe that waveguides offer the best combination of strong coupling, high power handling capability, maximum use of active length and natural rejection of the fundamental mode. We use multiple waveguides at both ends of the cavity to captures any orientation of dipole modes and all monopole modes even if the field profile is tilted after tuning. The waveguide end groups at opposite ends of the cavity are offset in azimuth, figure 2, to allow high-order multipoles to be captured and to allow a straight run for the helium header and fill lines along the module. Figure 3 shows the broad-band monopole spectrum for this cavity calculated by MAFIA and the high-current spectral lines for $1 \mathrm{~A}, 750 \mathrm{MHz}$ recirculated beam. Figure 4 shows the dipole spectrum.

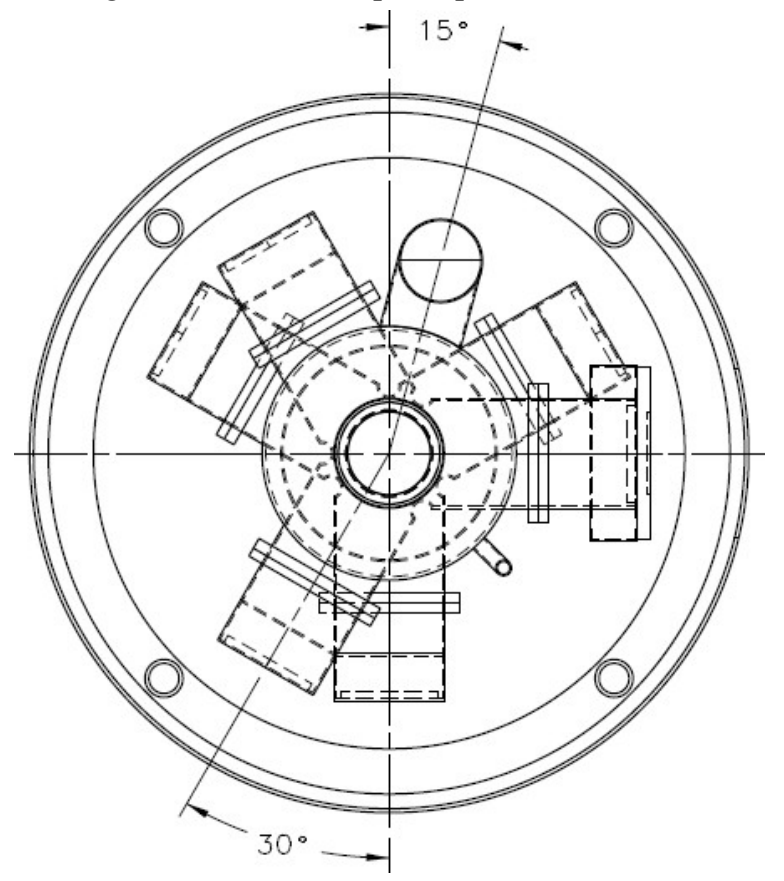

Figure 2. End view showing angular offset of HOM loads and two-phase helium header line.

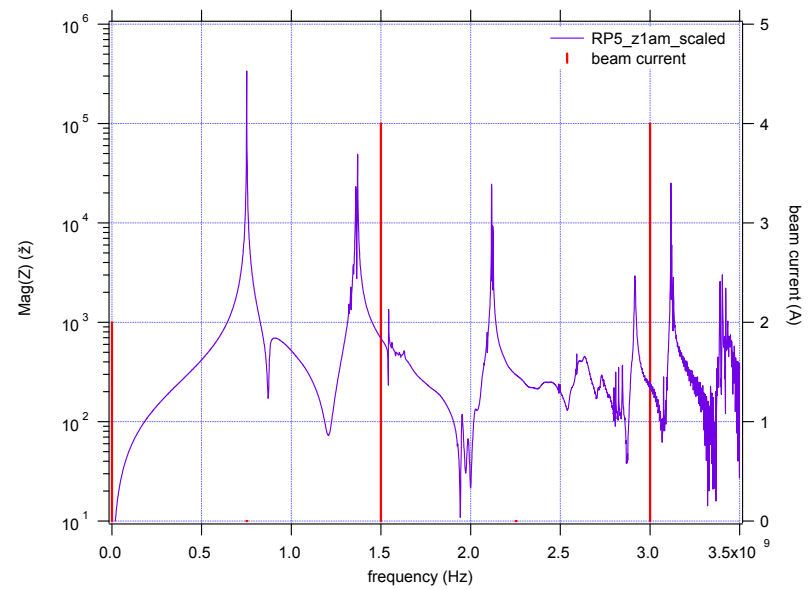

Figure 3. 5-cell rounded pillbox monopole spectrum.

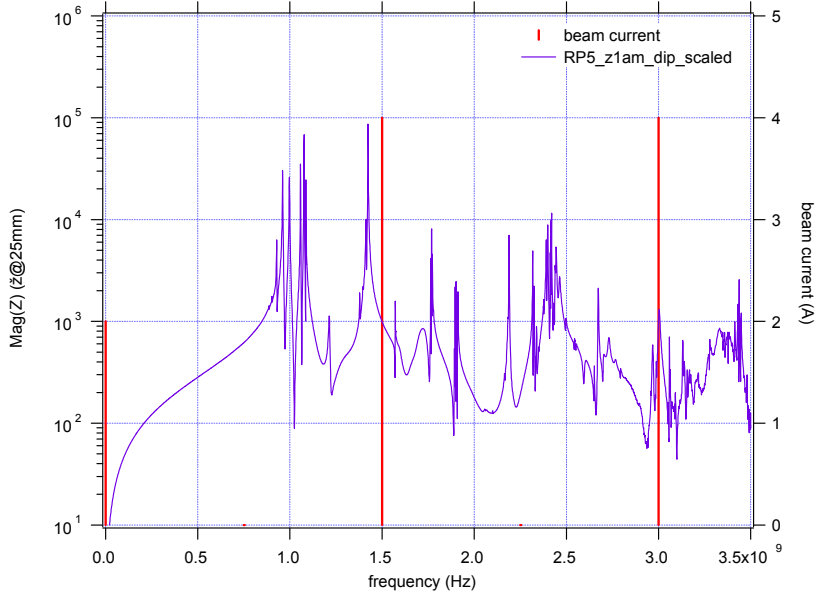

Figure 4. 5-cell rounded pillbox dipole spectrum

\section{Window and fundamental power coupler}

For the high-current ERL the beam power required could be significant if the machine is operated with less than full energy recovery, for example to supply up to $1 \mathrm{MW}$ optical power from the linac RF system instead of the injector. For six cavities each fundamental power coupler would be required to supply about $167 \mathrm{~kW}$. This is well within the capability of existing coupler designs such as the PEP-II type waveguide window [3], figure 5, or SNS coaxial coupler [4], figure 6. In either case dynamic losses in the warm section will be intercepted at $50 \mathrm{~K}$ but losses between that point and the cavity will contribute to the $2 \mathrm{~K}$ heat load. Trace cooling of the outer conductor will be employed and the location of the $50 \mathrm{~K}$ heat station will be determined to minimize the total operating load. If an SNS style coaxial coupler is used the center conductor will also be gas cooled.

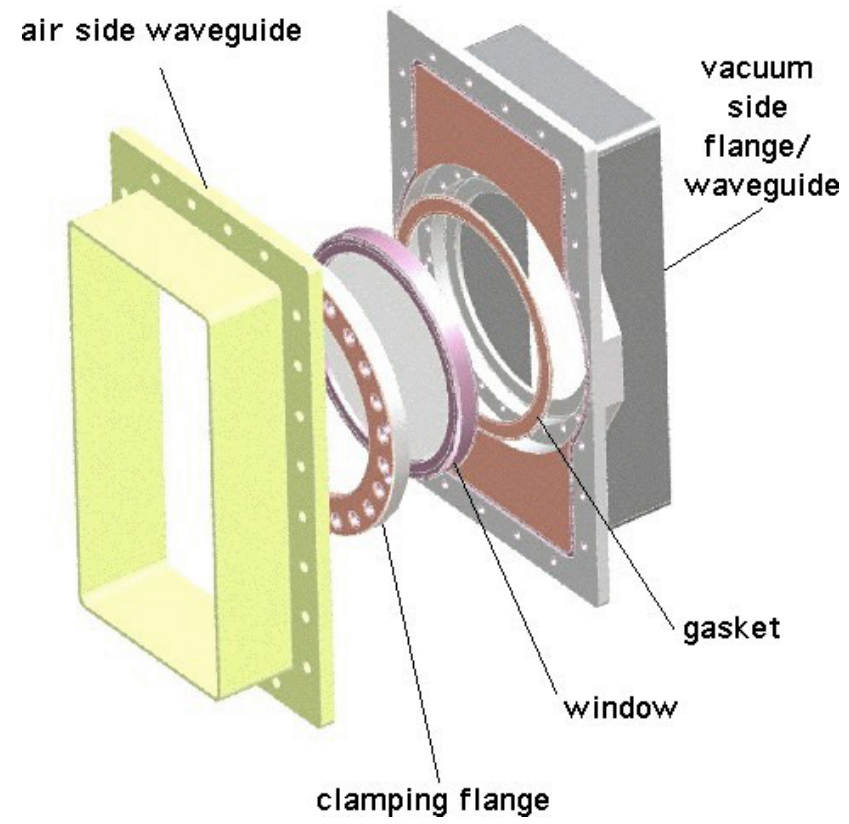

Figure 5. PEP-II type waveguide window assembly. 


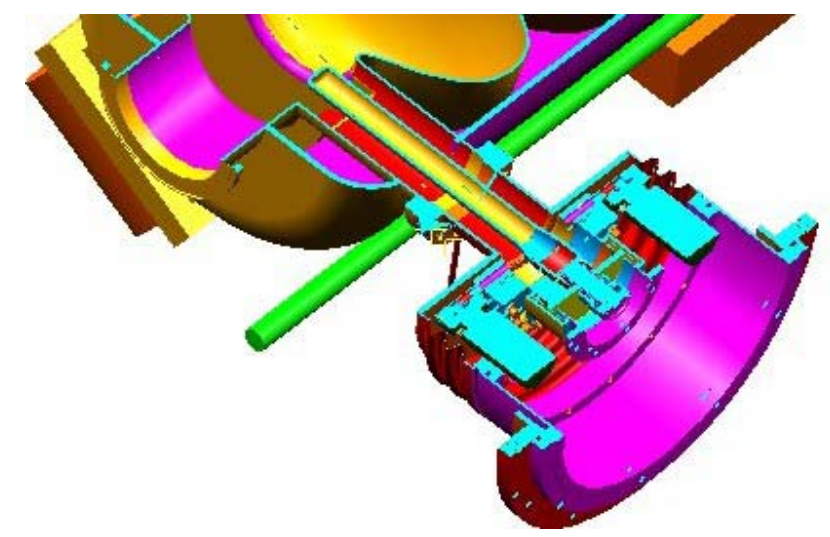

Figure 6. SNS-type coaxial coupler assembly.

\section{HOM load}

Placing the HOM loads at ambient temperature allows a wide choice of options for dissipating the power. We propose to use wedges of absorbing ceramic material in vacuum as used on the PEP-II cavities, see figure 7, [5]. This solution provides a high-power broad-band load using simple fabrication steps and commercially available materials, such as $\mathrm{AlN} / \mathrm{SiC}$, with good vacuum properties.

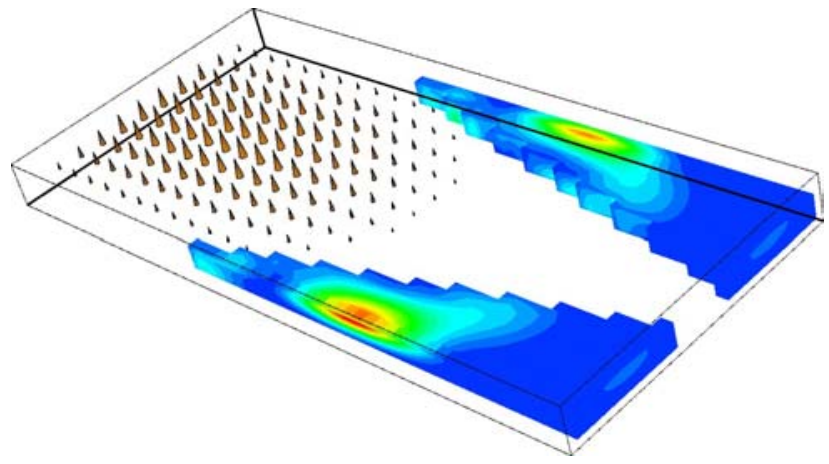

Figure 7. MAFIA model of broadband dielectric absorber.

\section{Tuner}

We propose to use a modified version of either the $12 \mathrm{GeV}$ upgrade style tuner mounted on the helium vessel, or SNS-style tuner [6], located on the end of the helium vessel. Detailed studies of these two configurations are ongoing. Real-estate constraints due to the number of dampers and the layout of the end groups may dictate the eventual solution. In either case the motor will be accessible for servicing.

\section{Integration}

We propose to follow the process employed at JLab for the SNS and upgrade prototype modules. Cavities will be pre-qualified and assembled into a hermetically sealed string under cleanroom conditions. The string will be transferred under vacuum to an assembly rail where ancillary components, magnetic and heat shields and thermal insulation will be added. A space frame will be assembled around the cold mass and the string will be suspended from it and aligned to it. The space frame assembly will then be inserted into a vacuum vessel and end cans added.

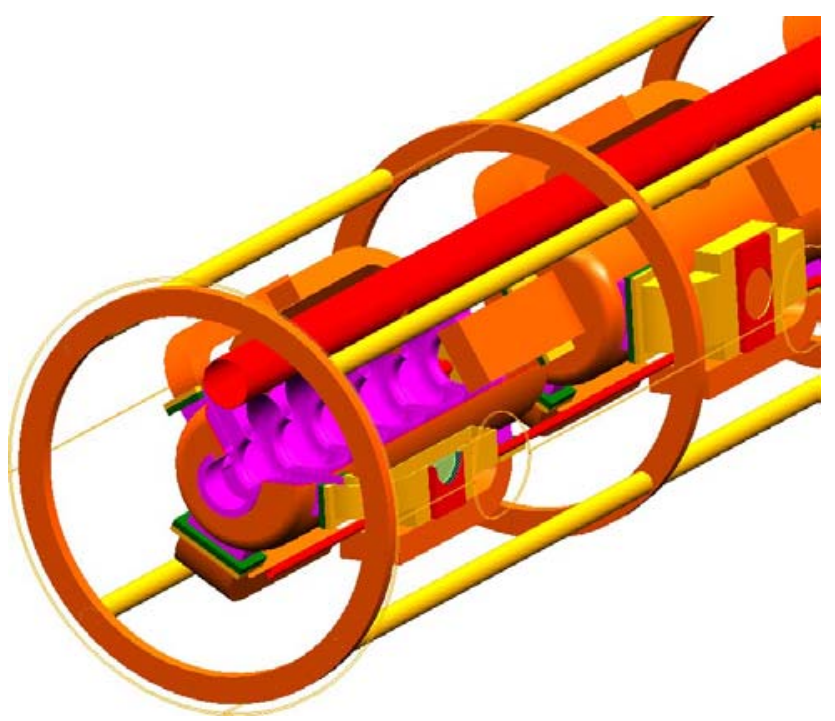

Figure 9. Cavity assembly in SNS-style space frame.

\section{TO DO LIST}

Multipacting analysis of the new cell shape is under way. Tasks remaining to be done include detailed thermal modeling, microphonic analysis of the structure and detailed design and prototyping of all main components. The goal, funding permitting, is to have a prototype cryomodule on the assembly rails by the end of FY 07 .

\section{CONCLUSIONS}

We have developed concepts for the key components of an Ampere-class cryomodule for high-power FEL and other applications. Detailed study shows that it is possible to achieve the HOM damping required in a multi-cell cavity with while preserving good fundamental mode efficiency and real-estate gradient.

Concepts for the module layout and ancillary components are closely based on existing proven designs and production methods and processes in use at JLab.

\section{REFERENCES}

[1] R. Rimmer et. al., "Strongly HOM-damped Multi-cell RF Cavities for High-Current Applications," SRF03, Lubeck, Germany (2003)

[2] H. Wang et. al., "Elliptical Cavity Shape Optimization for Acceleration and HOM Damping," these proceedings.

[3] R.A. Rimmer et. al., "A High-Power L-Band RF Window," PAC'01, Chicago, 2001.

[4] M. Stirbet et. al., "RF Conditioning and Testing of Fundamental Power Couplers for SNS Superconducting Cavity Production," these proceedings.

[5] R. Pendleton et. al., "PEP-II B-Factory Prototype Higher Order Mode Load Design," PAC 95, Dallas, 2005.

[6] J. Preble et. al., "SNS Cryomodule Performance,", PAC 2003, Portland, USA. 